\title{
Uma Estratégia de Encaminhamento Eficiente para Redes de Veículos Aéreos não Tripulados de Dados Nomeados
}

\author{
Francisco R. C. Araújo ${ }^{1}$, Antonio M. de Sousa ${ }^{1}$, Leobino N. Sampaio ${ }^{1}$ \\ ${ }^{1}$ Programa de Pós-Graduação em Ciência da Computação (PGCOMP) \\ Departamento de Ciência da Computação - Universidade Federal da Bahia (UFBA) \\ Salvador - BA - Brasil \\ \{franciscorca, antonio.mateus, leobino\}@ufba.br
}

\begin{abstract}
Named Data Networking (NDN) has emerged as a suitable solution for the current challenges faced by IP architecture. Although NDN is a promising paradigm, some challenges need to be addressed, especially in wireless and mobile environments. Such scenarios suffer from packet flooding that degrades the network performance. In this work, we propose a forwarding strategy for Named Data Mobile Ad hoc Networks that aims to mitigate the uncontrolled flooding. The Interest and Data packets update the level of uncertainty for each node according to the retrieval of content. Besides, nodes also consider the signal strength received by the interface at the time of Interest entry as a forwarding criterion. We analyze our strategy in the Flying Ad hoc Networks environment, and the simulations results show gains in the reduction broadcast storm while maintaining higher interest satisfaction rate.
\end{abstract}

\begin{abstract}
Resumo. As Redes de Dados Nomeados (NDN) foram propostas para lidar com os atuais desafios enfrentados pelas redes IP tradicionais. A NDN, embora promissora, aplicada aos ambientes sem fio e de mobilidade sofre com a inundação de pacotes e a sobrecarga gerada. Neste trabalho é proposta uma estratégia de encaminhamento para Redes Ad hoc Móveis de Dados Nomeados que visa reduzir a inundação de pacotes. O fluxo de pacotes de interesse e dados atualiza o nível de incerteza de cada nó em relação à recuperação de conteúdos. Além disso, os nós também consideram a intensidade de sinal recebido pela interface no momento do ingresso do interesse como critério de encaminhamento. A avaliação se deu num ambiente de Redes de Veículos Aéreos não Tripulados e os resultados de simulações mostram ganhos na redução do broadcast storm, enquanto mantém uma elevada taxa de satisfação de interesse.
\end{abstract}

\section{Introdução}

As redes de veículos aéreos não tripulados (do inglês, Flying Ad hoc Networks - FANETs) consistem em um tipo de rede ad hoc móvel (do inglês, Mobile Ad hoc Network - MANET) caracterizada por dispositivos que possuem reduzido poder computacional e energético, alta mobilidade dos nós e conexões intermitentes entre os mesmos. Em razão de tais características, as FANETs sofrem diversos desafios e muitos deles estão associados às limitações da arquitetura IP. Dessa forma, a arquitetura de redes de dados nomeados (do inglês, Named Data Networking - NDN) [Zhang et al. 2014] surge como uma atraente alternativa para a implementação de FANETs (FNDN) [Barka et al. 2018] objetivando endereçar parte das questões em aberto nessas redes. 
A NDN em ambientes ad hoc [Liu et al. 2017] é uma tendência recente. Trata-se de uma arquitetura centrada na informação que permite desvincular o conteúdo de sua localização, em vez de associá-lo ao endereço IP como empregado nas redes atuais. A arquitetura também possui cache na camada de rede, além de permitir entrega multicast e controle de fluxo naturalmente. Apesar desses benefícios, em ambientes de NDN sob MANET, é praticamente inviável manter a FIB (do inglês, Forward Information Base) dos nós dada a alta dinamicidade dessas redes [Grassi et al. 2014]. Além disso, na NDN, os dados retornam para os consumidores através da trilha deixada pelos interesses (i.e., pacotes que recuperam dados) na PIT (do inglês, Pending Interest Table) dos nós ao longo do caminho percorrido até a fonte de dados. No entanto, em MANETs, os nós geralmente possuem apenas uma interface de camada física [Yu et al. 2013], além do próprio meio físico que gera vários desafios para a comunicação. Dessa forma, a comunicação nesses ambientes acontece por meio da difusão de pacotes, o que torna inevitável o problema do broadcast storm [Amadeo et al. 2015] e seus efeitos colaterais, tais como o tráfego de pacotes redundantes, as colisões e o desperdício de recursos de rede e dos nós [Liu et al. 2017]. Tais problemas tornam-se ainda mais evidentes em ambientes de FANETs, uma vez que o escopo de mobilidade dos nós é mais amplo por isso, existe uma maior incerteza sobre o sucesso da obtenção de conteúdos na rede.

Diante das questões apresentadas, grande parte dos trabalhos da literatura que investigou tais questões concentram-se em reduzir os interesses redundantes retransmitidos, uma vez que a NDN possui um modelo de comunicação reativo. De forma geral, há duas principais correntes de ideias disponíveis: (i) reduzir a quantidade de nós capazes de encaminhar o interesse [Ahmed et al. 2015, Grassi et al. 2015, de Sousa et al. 2018, Barka et al. 2018] e assim diminuir os interesses encaminhados ou atacar diretamente a redução da quantidade de interesses encaminhados em cada nó [Yu et al. 2013]; e (ii) permitir a livre retransmissão de interesses e tentar reduzir a quantidade de dados redundantes retornados [Ahmed et al. 2016a, Ahmed et al. 2016b]. No entanto, esses trabalhos não aproveitam as características da própria NDN, e introduzem novas estruturas à arquitetura ou alteram as existentes, além de gerar carga extra de controle, utilizando técnicas da rede IP e contrárias à NDN.

Este trabalho apresenta, portanto, uma estratégia de encaminhamento de interesse para FNDN que visa reduzir o broadcast storm e seus danos. A solução consiste em proporcionar que cada nó tenha conhecimento de suas chances de recuperação do conteúdo solicitado. De acordo com o nível de chances da recuperação do conteúdo, o nó consegue tomar decisões localmente sobre o encaminhamento ou descarte do interesse recebido. As decisões são baseadas no nível de incerteza que o nó possui para o prefixo de nome do interesse, bem como na intensidade de sinal recebido pela interface de entrada do interesse no momento de seu ingresso. O diferencial da proposta está em utilizar a própria carga da rede para ajustar a ciência da recuperação de conteúdo em cada nó, evitando a geração de tráfego de controle, além de não alterar nenhum componente da arquitetura da NDN nem adicionar novos. A estratégia é totalmente dinâmica e ajustável de acordo com o fluxo de pacotes, além de intrínseca aos conceitos da NDN. Ademais, a estratégia pode reduzir os efeitos provocados por ataques de interesses falsos, uma vez que os nós se baseiam no seu nível de incerteza para alcançar um determinado conteúdo antes de encaminhar um interesse na rede. Ou seja, interesses falsos terão naturalmente um alto nível de incerteza, pois não poderão recuperar dados. 
Para comprovar os benefícios da proposta foram realizados experimentos por meio de simulação utilizando o simulador ndnSIM [Mastorakis et al. 2017]. O estudo consistiu em variar a quantidade de veículos aéreos não tripulados (do inglês, Unmanned Aerial Vehicles - UAVs), comparando o desempenho da proposta com a estratégia CONET [Ahmed et al. 2016a] e a estratégia Multicast [Mastorakis et al. 2017] nativa da NDN. Os resultados obtidos mostraram que a proposta consegue reduzir o broadcast storm enquanto mantém uma elevada taxa de satisfação de interesse.

O restante do trabalho está organizado da seguinte forma: na Seção 2 são apresentados os trabalhos relacionados e na Seção 3 a proposta é detalhada. Os experimentos e resultados são discutidos na Seção 4 e, por fim, o trabalho é concluído na Seção 5.

\section{Trabalhos Relacionados}

Nos últimos anos diversos trabalhos têm investigado formas de minimizar o problema do broadcast storm em MANETs. Devido ao modelo de comunicação reativo da NDN, a maioria dos trabalhos foca na redução da quantidade de nós aptos a reencaminhar o interesse para evitar a inundação de pacotes na rede.

Em [Ahmed et al. 2015] foi proposta uma estratégia de encaminhamento que seleciona apenas um nó entre os vizinhos do nó atual para reencaminhar os interesses do consumidor. A estratégia consiste na troca de informações sobre estatísticas de interesses satisfeitos entre os nós vizinhos, os quais contam com uma estrutura extra para armazenar essas informações. Além disso, cada nó mantém uma segunda estrutura com suas próprias estatísticas de interesses que foram satisfeitos. Já a estratégia de [Grassi et al. 2015] explora as informações de geolocalização para guiar os interesses em direção aos dados. Dessa forma, os autores desenvolveram mecanismos para mapear os nomes dos dados para a localização dos mesmos. A estratégia conta com o auxílio das unidades de acostamento nas rodovias e com a cache dos nós, os quais precisam utilizar o GPS (do inglês, Global Positioning System) para encontrar suas localizações geográficas.

No trabalho [de Sousa et al. 2018] foi proposta uma estratégia de encaminhamento baseada na velocidade e posição do nó. Os nós intermediários que recebem um interesse decidem enviá-lo ou descartá-lo, de acordo com sua distância atual, em relação ao nó que enviou o interesse, e sua velocidade. Dessa forma, como em [Grassi et al. 2015] os nós necessitam utilizar as coordenadas do GPS, além de não considerar possíveis interferências no sinal sem fio. O trabalho de [Yu et al. 2013] apresenta uma estratégia que elege o encaminhador do interesse baseado nas estatísticas de encaminhamento. Os nós decidem localmente encaminhar ou descartar um interesse, de acordo com a taxa de recuperação de dados do seu prefixo de nome. Para manter as estatísticas atualizadas, os nós coletam passivamente as taxas de pacotes encaminhados, no entanto, os nós que possuem uma baixa taxa de recuperação de dados periodicamente emitem sondas na rede para obterem uma atualização.

O trabalho de [Barka et al. 2018] é pioneiro por abordar a temática de FNDN. Os autores propuseram uma estratégia de encaminhamento voltada principalmente à segurança nesses ambientes e, como consequência, minimizam o broadcast storm de pacotes de interesse. Contudo, para alcançar os objetivos são realizadas algumas modificações na arquitetura da NDN, como a adição de tabela de vizinhos, identificação de nós e mudança no pacote de dados. Tais alterações devem ser evitadas, quando 
possível, pois vão contra o princípio da NDN de manter a centralidade no conteúdo.

Oposto aos trabalhos anteriores, os objetivos de [Ahmed et al. 2016a, Ahmed et al. 2016b] são voltados diretamente para o controle dos dados retornados, ao invés de reduzir os interesses retransmitidos. Dessa forma, os autores propuseram inserir um contador de saltos (do inglês, hop count) nos interesses que a cada novo salto é incrementado pelo nó encaminhador e salvo na entrada PIT correspondente antes de o interesse ser reencaminhado. Ao chegar no produtor ou em algum nó que possua o conteúdo em cache o valor do hop count presente no interesse é passado ao pacote de dados. De forma análoga, os dados retornam ao consumidor e a cada salto o hop count é decrementado. Se o hop count presente no dado for menor ou igual ao valor salvo na PIT do nó intermediário o dado é encaminhado, caso contrário é descartado.

Diferente das soluções apresentadas, que alteram os pacotes de interesse e dados [Ahmed et al. 2016b, Ahmed et al. 2016a], geram carga extra na rede [Yu et al. 2013, Ahmed et al. 2015], necessitam de informações auxiliares [Grassi et al. 2015, de Sousa et al. 2018] e adicionam novas estruturas à NDN [Barka et al. 2018, Ahmed et al. 2015], a presente estratégia foi projetada para se adequar totalmente à arquitetura NDN sem a necessidade da adição ou alteração de qualquer componente da mesma.

\section{Solução Proposta}

A estratégia proposta tem como objetivo principal reduzir o número de interesses encaminhados na rede sem degradar a satisfação de interesses, de modo a manter níveis adequados de dados recuperados. As próximas subseções apresentam detalhes de seu funcionamento.

\subsection{Visão Geral}

Para cumprir seus objetivos, a estratégia faz com que os nós tomem decisões ao realizar o reencaminhamento de um interesse na rede. Dessa forma, o nó deve julgar se é capaz de alcançar o conteúdo solicitado e se sua conexão, com o nó anterior que o encaminhou o interesse, é suficiente para que o mesmo possa retornar o dado solicitado. Portanto, o nó deve ter conhecimento do seu nível de incerteza (do inglês, Level of Uncertainty - LU), em relação à probabilidade de alcançar o conteúdo, e também conhecer a situação do indicador de intensidade de sinal recebido (do inglês, Received Signal Strength Indicator - RSSI) [Bohidar et al. 2015], na sua interface no momento que o interesse ingressou na mesma, para estimar o estado do enlace.

A Figura 1 ilustra o comportamento dos nós ao usar a estratégia proposta. $\mathrm{Na}$ figura há cinco nós encaminhadores (i.e., $E 1$ a $E 5$ ), um consumidor e um produtor (i.e.,

$C$ e $P$, respectivamente). É possível observar que $C$ ao enviar um interesse, todos os seus vizinhos o recebem (passos de 1 a 4) e em vez de simplesmente reencaminhá-lo, como faz a NDN nativa, os nós verificam a possibilidade de alcançar o conteúdo. Em reação ao passo 1, $E 1$ encaminha o interesse; após o passo 2, $E 2$ também o faz e $E 1$ irá apenas adicionar o pedido de $E 2$ em sua PIT, isso reflete o comportamento padrão da rede. No entanto, $\log$ o após o passo $3, E 3$ não encaminhará o interesse uma vez que possui um alto LU em relação ao conteúdo, algo semelhante ocorre em $E 4$ que também não encaminhará devido à baixa intensidade do sinal recebido. Dessa forma, os nós conseguem fazer um encaminhamento inteligente de pacotes evitando a inundação na rede. 


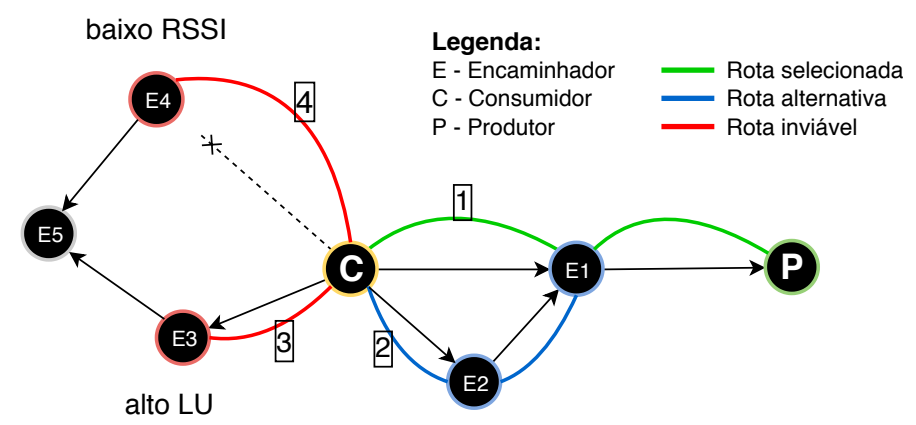

Figura 1. Encaminhamento ciente da recuperação de conteúdo.

Na próxima seção é apresentada em detalhes a decisão de encaminhamento tomada pelos nós da rede sempre que recebem um interesse.

\subsection{Decisão de Encaminhamento}

A Equação 1 representa o processo da tomada de decisão de encaminhamento do interesse $i$, em um determinado nó, para a sua interface de saída $j, F(i, j)$. Tal decisão é definida pela média ponderada dos fatores: (a) nível de incerteza e (b) indicador de intensidade de sinal recebido. O LU pode variar no intervalo de $[1,10]$, já o RSSI é considerado na equação com um intervalo de $[1,100]$. Tais fatores têm influências semelhantes no processo de decisão de encaminhamento, contudo, considerando uma escala de 1 a 10 o RSSI recebe um peso levemente maior que a média (i.e., $w_{r s s i}=6$ ), uma vez que representa o estado da conexão entre dois nós, enquanto o LU possui um peso aproximado (i.e., $w_{l u}=4$ ) dada sua significância para o sistema.

$$
F(i, j)=\frac{\left(L U(i, j) \times 10 \times w_{l u}\right)+\left(|R S S I| \times w_{r s s i}\right)}{100}
$$

A Equação 1 tem como restrição as Equações 2 e 3. Dessa forma, seu retorno sempre terá um valor de $0 \leq F(i, j) \leq 6$. Na Equação 2 foi considerado como aceitável os valores que variam entre o limite mínimo e a média $\mu$ do $L U$ (i.e., $1 \leq L U \leq 5$ ). Os valores maiores que $\mu$ representam rotas inviáveis para a recuperação de dados.

$$
f(L U)= \begin{cases}L U \leq \mu & \text { se } L U \text { é aceitável } \\ L U>\mu & \text { se } L U \text { não é aceitável }\end{cases}
$$

Na Equação 3, o valor da sensibilidade de recepção de sinal $\bar{x}$ foi definido igual a $-80 \mathrm{dBm}$, semelhante ao trabalho de [Amadeo et al. 2015], valores abaixo de $\bar{x}$ representam conexões instáveis.

$$
f(R S S I)= \begin{cases}R S S I \geq \bar{x} & \text { se } R S S I \text { é aceitável } \\ R S S I<\bar{x} & \text { se } R S S I \text { não é aceitável }\end{cases}
$$

O nível de incerteza de um UAV é determinado dinamicamente pelo fluxo de pacotes de interesse e dados trafegados no nó. A Equação 4 define o cálculo do LU em 
relação ao prefixo de nome do interesse $i$ para sua interface de saída $j$. É possível observar que os pacotes de interesse que não conseguem alcançar o conteúdo solicitado, isto é, os interesses expirados da PIT, elevam o LU do prefixo do interesse solicitado. Por outro lado, os pacotes de dados que conseguem retornar ao nó e os interesses satisfeitos pela CS (do inglês, Content Store) diminuem o seu nível de incerteza.

$$
L U(i, j)=i_{\text {Expirado }}-\left(d_{\text {Recebido }}+d_{\text {emCS }}\right) ; \quad \text { onde: }
$$

- $i_{\text {Expirado }}$ - é o interesse que foi encaminhado na rede, mas devido a não obtenção dos dados correspondentes, enquanto aguardava na PIT do nó pela chegada dos mesmos, alcançou sua duração máxima estipulada e foi removido (i.e., atingiu seu tempo de vida);

- $d_{\text {Recebido }}$ - é o pacote de dados que retorna ao nó, após uma solicitação através de um interesse;

- $d_{e m C S}$ - é o pacote de dados armazenado na CS do nó, o qual pode satisfazer o interesse na cache, caso o nó a possua.

\subsection{Interação com a Arquitetura NDN}

A estratégia de encaminhamento proposta intrinsecamente utiliza os principais componentes da arquitetura da NDN, o que dispensa qualquer modificação na arquitetura. Tais componentes são: a PIT, usada para manter os registros dos interesses enviados para possibilitar que os dados recuperados possam retornar ao consumidor; a FIB, que gerencia as interfaces de saída para encaminhar os interesses; e a CS que é capaz de armazenar dados [Zhang et al. 2014]. A estratégia, portanto, atua de forma conjunta com os elementos arquiteturais da rede, como descrito a seguir:

- FIB - utilização dessa estrutura para armazenar as informações do estado atual da rede. A FIB tem sua função bastante limitada nas redes ad hoc móveis de dados nomeados, pois a dinâmica da conectividade entre os nós em movimento inviabiliza a execução de um protocolo de roteamento que mantenha a FIB atualizada [Grassi et al. 2014]. Como pode ser observado na Figura 2, a FIB é composta de entradas com seus respectivos prefixos ou rotas, onde cada entrada possui ao menos uma interface de saída e um custo atrelado a mesma. O custo é atualizado pelo protocolo de roteamento ${ }^{1}$, e na falta de um protocolo esse campo fica inativo. Portanto, a estratégia proposta estende as funcionalidades da FIB para manter o LU atual do prefixo no campo custo em cada nó e, dessa forma, evita a adição de estruturas extras ou a modificação na arquitetura.

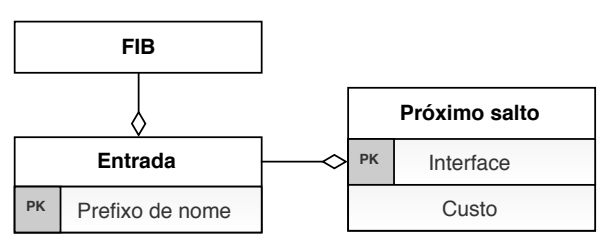

\begin{tabular}{|l|l|l|}
\hline \multicolumn{3}{|c|}{ FIB } \\
\hline Prefixo & Interfaces & Custo \\
\hline /temperatura & 1 & 1 \\
\hline /umidade & 1 & 8 \\
\hline /video2 & 1 & 3 \\
\hline
\end{tabular}

Figura 2. Relacionamento de componentes e preenchimento da FIB.

\footnotetext{
${ }^{1}$ NDN, Technical Report NDN-0021. Disponível em: http://named-data.net/ techreports.html.
} 
- PIT - utilização para registro dos interesses que expiram o tempo de vida. Tais interesses contribuem para o aumento do nível de incerteza do nó na obtenção dos dados solicitados;

- CS - quando habilitada no nó, pode contribuir para baixar o LU do mesmo. Uma vez que se gera a possibilidade de os interesses serem satisfeitos em cache;

- Pacotes de interesse - utiliza os interesses trafegados na rede para atualizar o LU do nó. De modo que, os interesses que não atendem a Equação 1 não são encaminhados e consequentemente são descartados o que contribui diretamente com o aumento do LU do nó em relação ao prefixo do conteúdo solicitado. Da mesma forma, os interesses que foram encaminhados na rede e que não obtiveram resposta de dados, durante sua permanência na PIT, contribuem para o aumento do nível de incerteza do nó para obter os dados solicitados. Uma vez que o limite superior do LU do prefixo de nome é atingido, o próximo interesse expirado correspondente, fará com que o nível de incerteza volte a receber seu limite mínimo. Dessa forma, inicia-se todo o processo de ajuste do nível de incerteza novamente; - Pacotes de dados - semelhante ao tratamento oferecido aos interesses, a solução utiliza os dados trafegados na rede para atualizar o LU dos nós. A diferença é que enquanto os interesses que não podem alcançar os conteúdos solicitados elevam o LU, os dados que conseguem chegar no nó diminuem o seu nível de incerteza no prefixo armazenado na FIB, habilitando o nó a encaminhar os interesses futuros correspondentes aos prefixos que possuírem um baixo LU na FIB.

Como pode ser observado, a interação da estratégia é mais forte com a FIB, a qual é atualizada constantemente pelo fluxo dos pacotes de interesse e dados trafegados no nó. Por outro lado, as estruturas PIT e CS interagem indiretamente com a solução.

\subsection{Funcionamento da Estratégia}

A estratégia de encaminhamento proposta é dividida em três funções principais, as quais são discutidas nas próximas subseções.

\subsubsection{Envio ou reencaminhamento de interesse}

O Algoritmo 1 define o conjunto de ações que são realizadas por um nó, logo após o mesmo receber um pacote de interesse. A entrada FIB correspondente é encontrada através de uma pesquisa com a entrada PIT passada ao algoritmo (linha 2). Em seguida, é realizada uma consulta por cada interface da FIB atrelada à rota, cujo prefixo seja uniforme ao nome do interesse recebido (linhas 3 a 9). Durante essa consulta é verificada a origem do interesse. Se o pacote veio da camada de aplicação local, significa que o próprio nó corrente gerou o interesse e deseja enviá-lo na rede, então é chamada uma função para o envio do mesmo (linhas 4 e 5).

Em contrapartida, se a origem do interesse difere do nó corrente, isso indica que o mesmo veio da rede (linhas 6 a 9). Dessa forma, o nó precisa decidir se reencaminha ou não, para a rede, o interesse recebido, de acordo com as rotas armazenadas em sua FIB. A estratégia proposta considera no processo de tomada de decisão de encaminhamento o valor do RSSI obtido da interface pela qual o interesse ingressou no nó, bem como, o valor do LU da entrada FIB correspondente ao prefixo de nome do interesse. Desse modo, 
para os casos em que os valores do RSSI e do LU são avaliados como sendo adequados, segundo o cálculo da Equação 1, o nó decide encaminhar o interesse na rede (linhas 8 e 9). Depois de realizada a pesquisa pelas entradas da FIB correspondentes ao interesse, é checado se o interesse foi encaminhado, caso não tenha sido o mesmo é removido da PIT do nó (linhas 10 e 11).

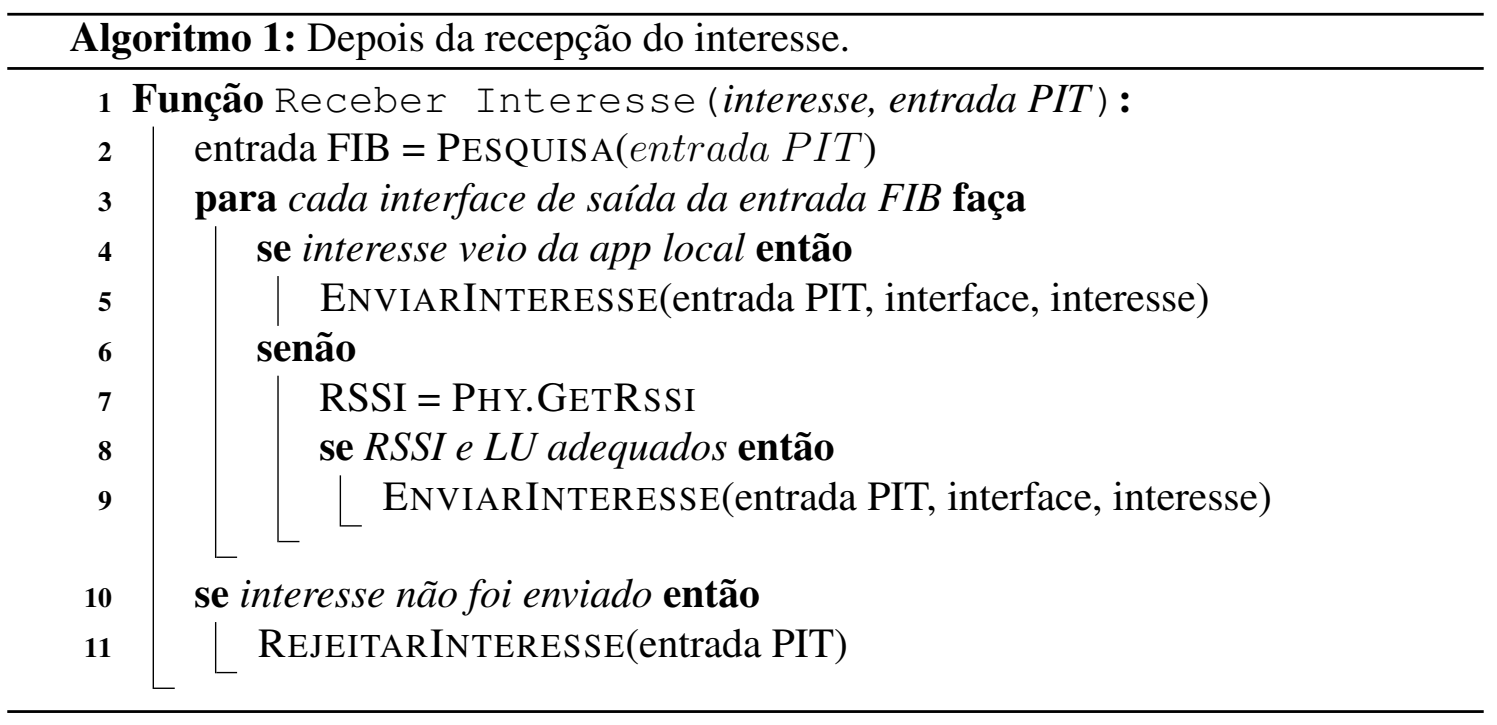

\subsubsection{Satisfação de interesse}

Para que um interesse possa ser resolvido ou satisfeito em um nó, há três possibilidades: (i) quando um pacote de dados que havia sido requisitado ingressa em uma de suas interfaces; (ii) quando um interesse pode encontrar o dado em cache; e (iii) quando o nó é próprio produtor do conteúdo solicitado. O Algoritmo 2 descreve o processo seguido em um determinado nó quando ele é capaz de resolver um interesse.

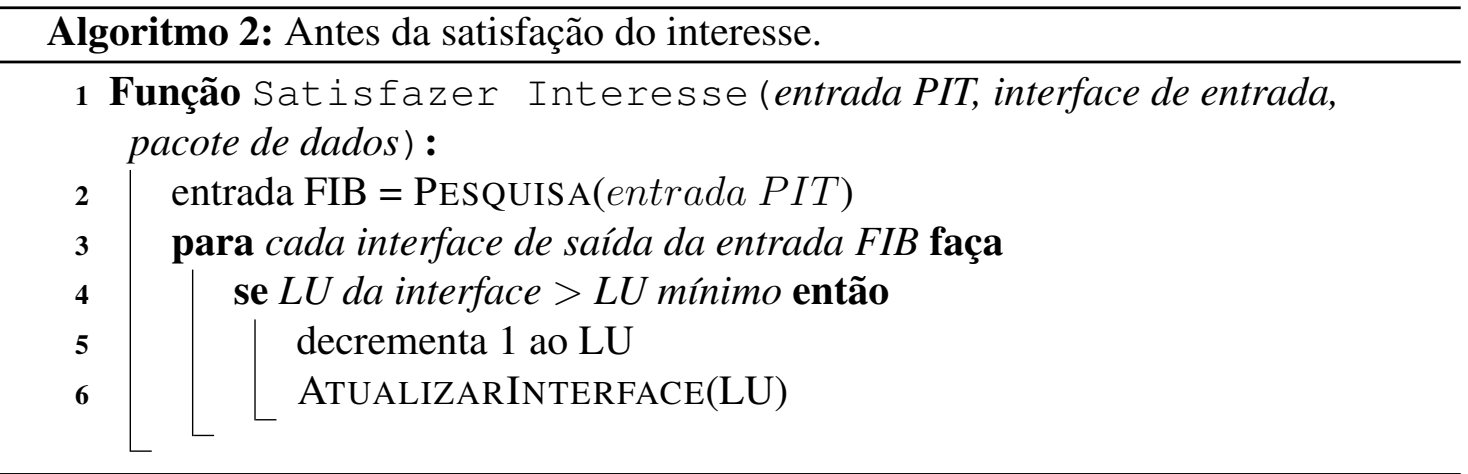

Como no algoritmo anterior, a entrada da FIB correspondente ao dado de entrada é buscada por meio da entrada PIT que armazena o interesse pendente para o dado (linha 2). Em seguida, é realizada uma iteração em cada interface da FIB que possui uma rota armazenada para o prefixo de nome equivalente ao nome do dado de entrada (linhas 3 a 6). Durante essa busca, é realizada uma checagem para atualizar o LU de cada rota da FIB correspondente ao dado (linhas 4 a 6). Dessa forma, quanto maior a capacidade de 
um nó de satisfazer interesses de um certo prefixo, menor será o LU da rota presente na FIB para o referido prefixo. Consequentemente, as rotas com baixo LU possibilitam ao nó encaminhar qualquer interesse compatível à rota.

\subsubsection{Expiração de interesse}

O Algoritmo 3 descreve os passos tomados quando um interesse alcança seu tempo máximo de permanência na PIT de um nó. Isso ocorre nos casos que o interesse foi enviado na rede e não alcançou o produtor do conteúdo ou um nó intermediário que possuísse uma réplica do dado solicitado em cache. Portanto, nenhum pacote de dados correspondente ao nome do interesse que ficou na PIT do nó no estado de espera, pode ser retornado o que provoca a remoção do interesse da entrada PIT. No algoritmo a entrada da FIB é conseguida após uma consulta com a entrada PIT (linha 2). Em seguida, é desempenhada uma iteração nas interfaces da entrada FIB que possuam rotas armazenadas com os prefixos de nome equivalentes ao nome do interesse prestes a expirar (linhas 3 a 8).

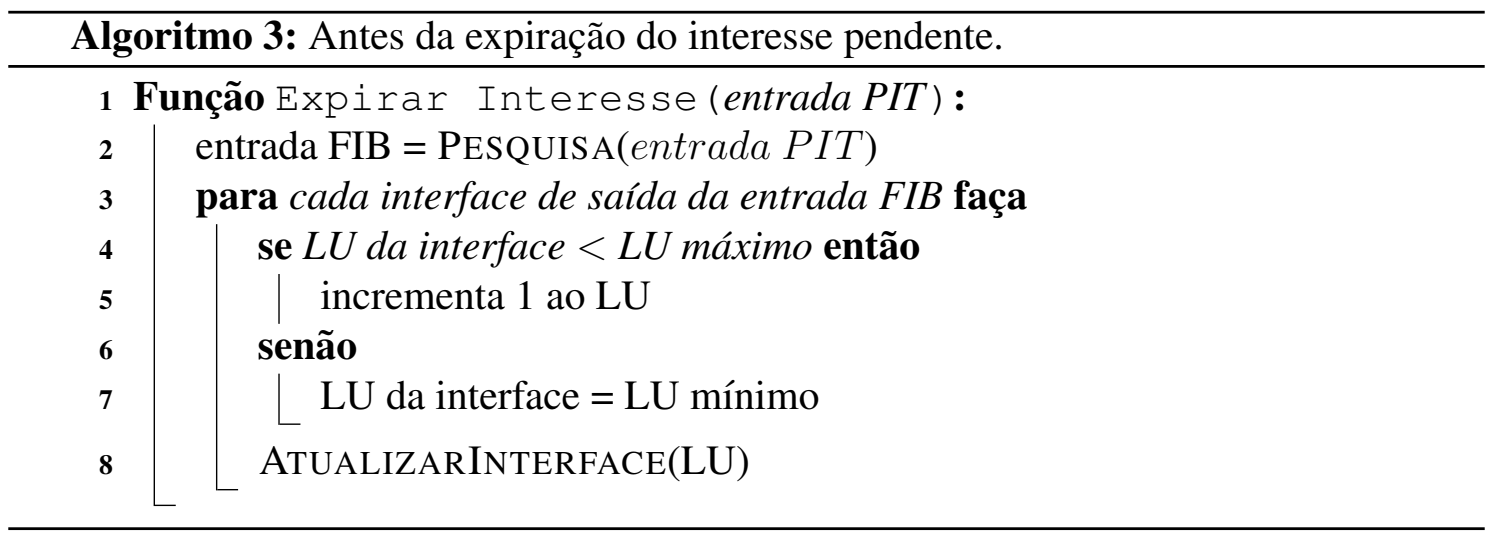

Durante a consulta pelas rotas relacionadas ao interesse é feita uma checagem para atualizar o LU de cada interface consultada. Para tal, é verificado se o LU da entrada FIB em questão não atingiu o seu limite máximo para que o seu valor possa ser aumentado (linhas 4 e 5). Em seguida, caso o LU tenha atingido o seu limite superior, o mesmo recebe o valor do seu limite inferior (linhas 6 e 7). Portanto, reiniciando o valor do LU do prefixo na interface, o que possibilita ao nó a retomada do encaminhamento para os interesses futuros, cujo prefixo seja comum às entradas FIBs com o valor do LU aceitável. Finalmente, o LU da rota relacionada à interface é atualizado na entrada FIB (linha 8).

\section{Estudo Experimental e Análise dos Resultados}

\subsection{Ambiente de Experimentação}

Para avaliar o desempenho da solução proposta, foi desenvolvido o ambiente de experimentação no simulador de redes, baseado em eventos discretos, ndnSIM [Mastorakis et al. 2017], o qual é um módulo do NS-32 específico para NDN.

$\mathrm{O}$ ambiente avaliado consiste em uma rede FNDN composta de UAVs. Cada UAV equipado com uma interface 802.11 p, se movendo através do modelo de mobilidade 3D

\footnotetext{
${ }^{2}$ https: / / www.nsnam.org/
} 
Random Waypoint (RWP), numa área de $2 \mathrm{~km}^{2}$ com até 30 metros de altitude, a uma velocidade que varia de 10 a $15 \mathrm{~m} / \mathrm{s}$ e com um alcance de transmissão de 400 metros [Barka et al. 2018]. A quantidade de UAVs seguiu uma progressão aritmética crescente finita P.A $(10,20,30,40,50,60)$ de razão $r=10$. A quantidade de consumidores e produtores assumiu $10 \%$ da quantidade de UAVs, respectivamente [Grassi et al. 2014]. Para cada simulação os consumidores e produtores foram escolhidos e distribuídos aleatoriamente, junto aos outros UAVs no cenário [Barka et al. 2018]. Cada consumidor requisitou 5 interesses por segundo até atingir um limite de 1000 interesses requisitados e um tempo total de simulação de 200 segundos [Ahmed et al. 2016a]. A cache dos UAVs foi desabilitada para evitar que os dados fossem recuperados nos nós intermediários e assim forçá-los executar a estratégia de encaminhamento para alcançar os produtores. Por fim, o tempo de vida dos interesses na PIT foi configurado para 4 segundos [Ahmed et al. 2016a]. A Tabela 1 sumariza todos os parâmetros empregados nos experimentos.

Tabela 1. Parâmetros da Simulação.

\begin{tabular}{ll}
\hline Parâmetro & Valor \\
\hline Modelo de rádio propagação & Nakagami \\
Modelo de mobilidade dos UAVs & RWP \\
Alcance de transmissão dos UAVs & 400 metros \\
Velocidade dos UAVs & 10 a $15 \mathrm{~m} / \mathrm{s}$ \\
Protocolo de enlace & IEEE $802.11 \mathrm{p}$ \\
Tamanho do pacote de dados & 1024 bytes \\
Dimensão do cenário $(\mathrm{x} \times \mathrm{y}) \times \mathrm{z}$ & $2 \mathrm{~km}^{2} \times 30 \mathrm{~m}$ \\
Taxa de transmissão de interesse & $5 \mathrm{I} / \mathrm{s}$ \\
Quantidade de UAVs & $10,20,30, \ldots, 60$ \\
Quantidade de consumidores e produtores & $10 \%$ da \#UAVs respectivamente \\
Conteúdos requisitados por consumidor & 1000 \\
Tempo de simulação para cada execução & 200 segundos \\
Tempo de vida do interesse & 4 segundos \\
\hline
\end{tabular}

Cada experimento foi replicado 10 vezes com diferentes sementes para obter a média com um nível de confiança ${ }^{3}$ de $95 \%$. A proposta foi comparada com a estratégia CONET [Ahmed et al. 2016a] e com a estratégia Multicast nativa da NDN. É importante destacar que para ambas as soluções comparadas foram replicadas as mesmas especificações do ambiente de simulação.

\subsection{Métricas de Desempenho}

$\mathrm{Na}$ análise de desempenho foram consideradas as métricas que refletem o estado da rede e dos consumidores especificamente, as quais são descritas a seguir:

- Taxa de satisfação de interesse - consiste na média de pacotes de interesse que puderam encontrar os dados solicitados possibilitando que esses dados retornassem aos consumidores satisfazendo os seus interesses pendentes;

\footnotetext{
${ }^{3}$ Utilização da distribuição $t$-student e $\alpha=0,05 \%$.
} 
- Atraso médio - representa a média dos atrasos, em segundos, que os interesses dos consumidores levam para recuperar os dados solicitados. Esse tempo pode incluir possíveis timeout e retransmissões de interesses;

- Tráfego de pacotes - corresponde as médias individuais dos pacotes de interesse e dados trafegados nos UAVs. A quantidade de interesses encaminhados influencia no número de dados trafegados, em razão do modelo de comunicação receiverdriven da NDN [Liu et al. 2017];

- Taxa de interesses expirados - equivale à média dos interesses que foram removidos, após terem atingido seu tempo de permanência na PIT dos UAVs. Isso ocorre quando o interesse é encaminhado e não consegue alcançar o respectivo dado;

- Quantidade de saltos - representa a média dos saltos tomados pelos pacotes de dados a partir dos produtores até alcançar os seus consumidores.

\subsection{Resultados e Discussões}

A média da taxa de satisfação de interesse está representada na Figura 3. É possível observar que a proposta sempre se mantém igual ou superior às estratégias comparadas. Nos cenários de pouca densidade na rede, 10 e 20 nós, a proposta se mantém semelhante a CONET. Nos casos que a densidade da rede é maior, 30 a 60 nós, a proposta se iguala a estratégia Multicast atingindo elevadas taxas de conteúdos recuperados. Nestes casos, a CONET obtém uma taxa de satisfação de interesse inferior, isso reflete exatamente o seu comportamento esperado, pois a CONET foi projetada para reduzir os dados redundantes retornados, no entanto, isso prejudica a recuperação de conteúdos.

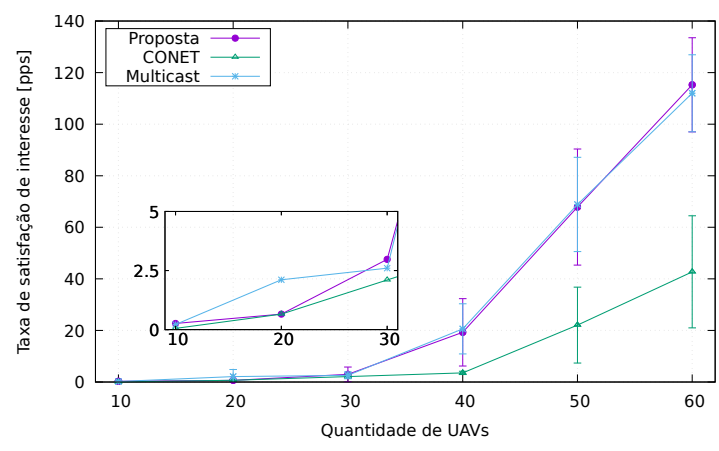

Figura 3. Taxa de satisfação de interesse.

O atraso médio dos interesses, que puderam recuperar os dados solicitados, está representado na Figura 4. Nota-se que à medida que o número de UAVs aumenta, o atraso também aumenta para as três estratégias avaliadas. Essa relação ocorre devido ao aumento da carga na rede, uma vez que a quantidade de consumidores equivale a $10 \%$ da quantidade total de UAVs. Como visto na Figura 3, o comportamento da proposta se mantém semelhante a CONET em ambientes menos densos e semelhante a Multicast em cenários de maior densidade. A CONET é a estratégia que obtém menor atraso, no entanto, como mostrado na figura anterior é a estratégia que satisfaz menos interesses.

A Figura 5 retrata o tráfego de pacotes de interesse e dados na rede. A Figura 5(a) mostra o tráfego de interesses. Nela, observa-se que a proposta consegue melhores resultados na redução da taxa de interesses trafegados, principalmente nos cenários de menor densidade na rede. À medida que a densidade aumenta, a taxa do tráfego de interesses 


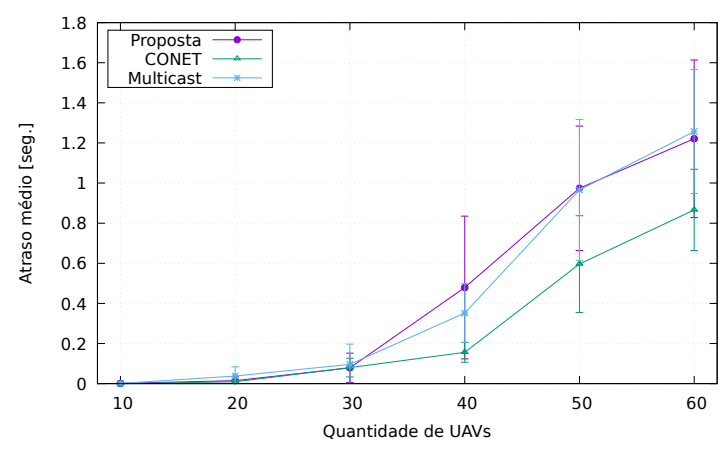

Figura 4. Atraso para recuperar dados.

se eleva, pois a carga aumenta com a quantidade de consumidores e nós encaminhadores aptos a reencaminhar os interesses recebidos. Além disso, com mais nós no cenário a possibilidade de um nó alcançar outro é maior, pois a área geográfica passa a ser melhor ocupada. Esta última condição faz com que o desempenho da proposta decresça, se aproximando do desempenho das estratégias comparadas, no entanto, ainda tendendo para menos interesses encaminhados. Por outro lado, a Figura 5(b) mostra a taxa de dados trafegados. Novamente a proposta se mantém semelhante a CONET quando há menos nós na rede e semelhante a Multicast quando a quantidade de nós é maior. Dessa forma, é possível observar que quando menos interesses são encaminhados, nos casos de 10 a 30 UAVs na Figura 5(a), a proposta obtém uma menor taxa de dados trafegados.

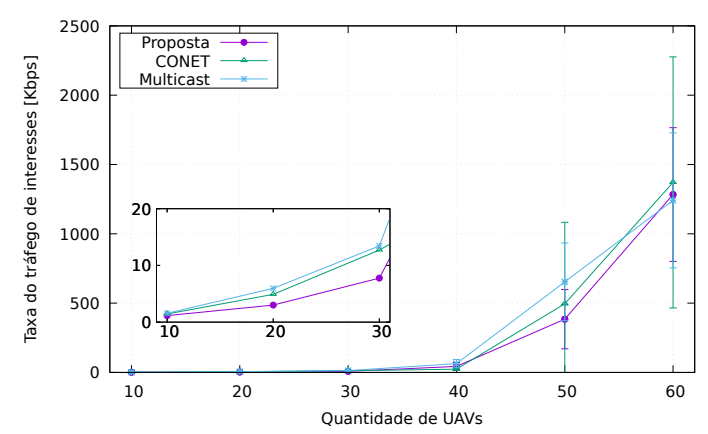

(a) Taxa do tráfego de interesses.

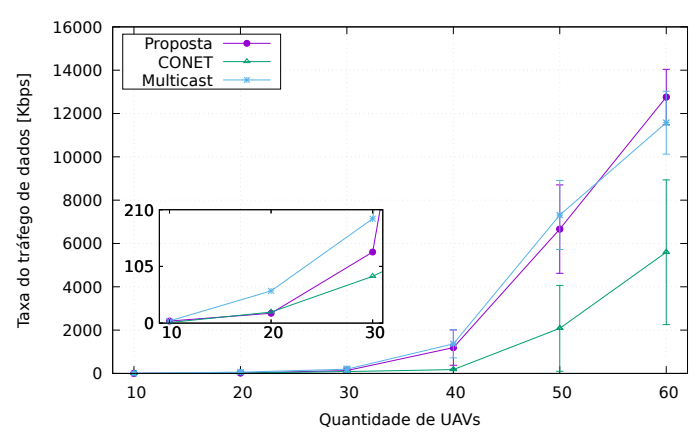

(b) Taxa do tráfego de dados.

Figura 5. Tráfego de pacotes na rede.

A Figura 6 ilustra a taxa de interesses que atingiram seu tempo máximo de permanência na PIT dos UAVs e consequentemente foram removidos. Isso ocorre quando os interesses enviados ou reencaminhados não conseguem alcançar os dados requisitados. $\mathrm{Na}$ figura, nota-se que a proposta mantém uma taxa de interesses expirados inferior as demais estratégias comparadas. Portanto, a proposta além de enviar menos interesses, possibilita aos que foram enviados recuperar os dados solicitados antes de atingirem seu tempo na PIT dos nós.

A média da quantidade de saltos tomados pelos dados para alcançar os consumidores, está representada na Figura 7. Nota-se que as três estratégias apresentam um comportamento similar. Ambas, no cenário com dez UAVs apresentam uma média geral inferior a um salto, devido à baixa densidade na rede o que dificulta os nós entrarem no 


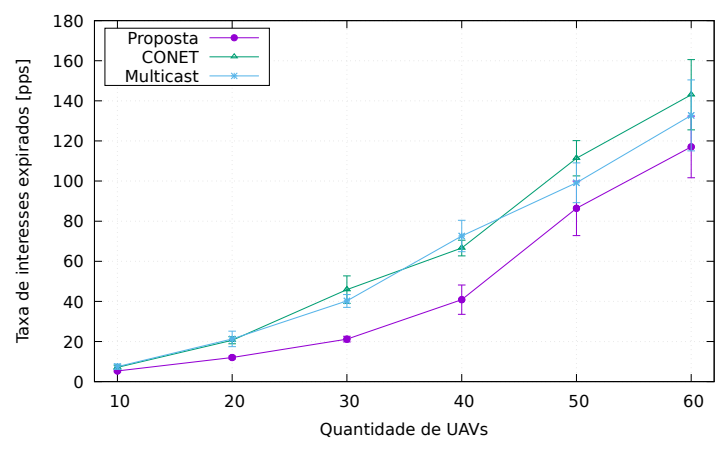

Figura 6. Taxa de interesses expirados.

raio de alcance de algum outro UAV. À medida que a densidade aumenta, há uma leve subida gradual da quantidade de saltos, pois as chances dos nós de entrarem no raio de alcance de algum vizinho são maiores. Dessa forma, as estratégias se mantêm semelhantes.

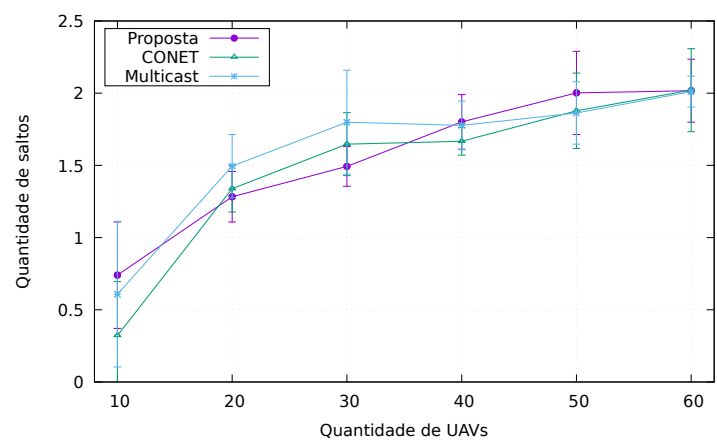

Figura 7. Quantidade de saltos dos dados recuperados.

\section{Conclusão e Trabalhos Futuros}

Em FANETs, problemas inerentes aos cenários de redes sem fio, relacionados à inundação de pacotes, ainda prevalecem mesmo quando se adota a arquitetura NDN para o encaminhamento de interesses. Neste trabalho propomos uma estratégia de encaminhamento para mitigar a inundação de pacotes na rede. A proposta consiste em proporcionar aos nós o encaminhamento ciente da recuperação de conteúdo, através da tomada de decisão baseada no nível de incerteza para obter o conteúdo e na intensidade de sinal recebido pela interface de entrada do interesse. Os resultados mostraram que a proposta mantém uma elevada taxa de satisfação de interesse em comparação com as estratégias avaliadas, reduz a quantidade de interesses encaminhados mitigando o problema do broadcast storm, além de apresentar uma menor taxa de interesses expirados na PIT dos nós, uma vez que mais dados podem ser recuperados antes da expiração do tempo de vida desses interesses. Além disso, a proposta se apresenta como uma estratégia de encaminhamento leve e intrínseca à NDN, pois a mesma não insere tráfego de controle, não altera nenhum componente da arquitetura da NDN e nem adiciona novos. Ainda, há fortes indícios que pode ser resiliente a ataques de negação de serviços por inundação de interesses falsos.

Como trabalho futuro pretende-se analisar o comportamento da estratégia sob efeito de ataque de inundação de interesses falsos para comprovar sua imunidade a tais ameaças, outra possibilidade é avaliar a proposta nos ambientes de VNDN. 


\section{Agradecimentos}

Os autores agradecem o apoio da CAPES e do CNPq.

\section{Referências}

Ahmed, S. H., Bouk, S. H., and Kim, D. (2015). RUFS: robust forwarder selection in vehicular content-centric networks. IEEE Communications Letters, 19(9):1616-1619.

Ahmed, S. H., Bouk, S. H., Yaqub, M. A., Kim, D., and Gerla, M. (2016a). CONET: controlled data packets propagation in vehicular named data networks. In 2016 13th IEEE Annual Consumer Communications Networking Conference (CCNC), pages 620-625.

Ahmed, S. H., Bouk, S. H., Yaqub, M. A., Kim, D., Song, H., and Lloret, J. (2016b). CODIE: Controlled Data and Interest Evaluation in Vehicular Named Data Networks. IEEE Transactions on Vehicular Technology, 65(6):3954-3963.

Amadeo, M., Campolo, C., and Molinaro, A. (2015). Forwarding strategies in named data wireless ad hoc networks: Design and evaluation. Journal of Network and Computer Applications, 50:148-158.

Barka, E., Kerrache, C. A., Hussain, R., Lagraa, N., Lakas, A., and Bouk, S. H. (2018). A Trusted Lightweight Communication Strategy for Flying Named Data Networking. Sensors (Basel, Switzerland), 18(8):1-18.

Bohidar, S., Behera, S., and Tripathy, C. R. (2015). A comparative view on received signal strength (RSS) based location estimation in WSN. In 2015 IEEE International Conference on Engineering and Technology (ICETECH), pages 1-7.

de Sousa, A. M., Araújo, F. R. C., and Sampaio, L. N. (2018). A Link-Stability-Based Interest-Forwarding Strategy For Vehicular Named Data Networks. IEEE Internet Computing, 22(3):16-26.

Grassi, G., Pesavento, D., Pau, G., Vuyyuru, R., Wakikawa, R., and Zhang, L. (2014). VANET via Named Data Networking. In 2014 IEEE Conference on Computer Communications Workshops (INFOCOM WKSHPS), pages 410-415.

Grassi, G., Pesavento, D., Pau, G., Zhang, L., and Fdida, S. (2015). Navigo: Interest forwarding by geolocations in vehicular Named Data Networking. In 2015 IEEE 16th International Symposium on A World of Wireless, Mobile and Multimedia Networks (WoWMoM), pages 1-10.

Liu, X., Li, Z., Yang, P., and Dong, Y. (2017). Information-centric mobile ad hoc networks and content routing: A survey. Ad Hoc Networks, 58:255-268.

Mastorakis, S., Afanasyev, A., and Zhang, L. (2017). On the Evolution of ndnSIM: An Open-Source Simulator for NDN Experimentation. ACM SIGCOMM Computer Communication Review, 47(3):19-33.

Yu, Y., Dilmaghani, R. B., Calo, S., Sanadidi, M. Y., and Gerla, M. (2013). Interest propagation in named data manets. In 2013 International Conference on Computing, Networking and Communications (ICNC), pages 1118-1122.

Zhang, L., Afanasyev, A., Burke, J., Jacobson, V., Claffy, K., Crowley, P., Papadopoulos, C., Wang, L., and Zhang, B. (2014). Named data networking. ACM SIGCOMM Computer Communication Review, 44(3):66-73. 